\title{
Bimanual multijoints coordination: A brief review
}

\author{
Yong $\mathrm{Li}^{*}$ \\ Department of Neurology, Klinikum rechts der Isar, Technische Universität München, Ismaninger Strasse 22, 81675 München, Germany
}

\begin{abstract}
Walking upright freed the hands for carrying and manipulating tools. This complex bimanual coordinative ability may have been an essential step to lead our ancestors' brains to grow. The current review aims to tackle the state-of-the-art control mechanisms in kinematics and motor control perspectives during performing bimanual multijoints coordinative movements. The endeavor is to delineate possible principles from task goals, manipulations and modules, related brain activation and connectivity perspectives, and benefits and limits of considered modules to apply these principles to improve the life quality of people with movement disorder diseases and people who want to be healthier.
\end{abstract}

About six million years ago, Sahelanthropus may have walked on two legs. Becoming bipedal, walking upright freed the hands for carrying and manipulating tools. This complex bimanual coordinative ability may have been an essential step to lead our ancestors' brains to grow. In daily life, the ability to master objects such as weapons, tools, stationaries etc. by strong and agile upper limbs is revolutionary throughout the human evolution.

The hierarchy of constraints in bimanual coordination: Bimanual multijoints coordinative activities have been studied at many levels of the cognitive and motor systems. Three paradigms, including drawing tasks with direct objectives using pens or styluses in both hands, e.g., circle [1-6], star-line, etc. [7-12], bimanual tasks with abstract temporal and spatial objectives employing manipulanda [13] and joy sticks [14], and freehand bimanual multijoints coordinative tasks have been utilized to study the representation of bimanual actions [15-17]. As a unique evolutional landmark, writing and drawing are mostly a unilateral task. Therefore, intensive training of non-dominant hand to handle a pen or stylus is often needed to minimize the unequal motor capability as compared to the skillful dominant hand. The other two paradigms well compromise the unequal skillfulness of both hands by applying unified devices or focusing on pure joint/limb movements. In the past few decades, research findings in bimanual coordination suggest a coalition of constraints in bimanual coordination. The identified constraints cover from cognitive or perceptual to neuromuscular level, which are centered by network-level perception-action interactions of the CNS [18]. Among these bimanual coordination constraints, the neuromuscular constraint is defined as the relative timing of homologous muscle activation. Relative phase $(\Phi)$, subtraction of the phase angle of each limb at each measurable time point during cyclic movement, serves as a measure of two-limb-coordination. In-phase motion (IN, $\Phi=0^{\circ}$ ) refers to moving the fingers, wrists, or forearms inwards and outwards from the body midline simultaneously activate homologous muscle groups. Anti-phase motion ( $\left.\mathrm{AN}, \Phi=180^{\circ}\right)$ means that homologous muscle groups are activated in alternation. In-phase is more stable than anti-phase in bimanual coordination. The higher the movement speed goes, the more differential stability between both coordination modes becomes [19,20]. These coordination modes are generic as they are evident across different movement effectors.
Directional constraint results in stable patterns when two-single-joints move in the same direction without muscle homology, for example, anti-phase motion in bimanual coordination. Another example is that iso-directional movement of elbow and wrist within an arm (ISO, simultaneous flexion and extension of elbow and wrist) is more stable than noniso-directional movement (NISO, elbow flexion with simultaneous wrist extension or vice versa). Our work in 2004 revealed that neuromuscular constraint is dominant over perceptual constraints by directly comparing two constraints within a study. In that study, healthy young volunteers performed well controlled bimanual wrist coordinative movement with a set of conditions composed by palmup or palm-down combinations [21]. Other constraints identified by iso- or multi-frequency patterns, different degrees in relative phase of coordinative complexity and movement amplitude, direction etc. all contribute to the coalition of constraints but are not main focus of this review therefore will not be reviewed in details.

To further conceptualize the coalition of constraints, two-singlejoint, bilateral or unilateral or within-limb, motions have been investigated thoroughly in the past decades. The information-processing and the dynamic-pattern framework have been discovered based on similar designs [18]. Under the information-processing framework, two-single-joint motions are considered as a special case of dual task that is interfered with limited neural resources. Many publications using bilateral two-single-joint paradigm show that the bimanual coordination network is a dynamic entity that changes as a function of task complexity (spatiotemporal interlimb relationships), difficulty level (e.g. performance speed), and experience (e.g. transferring unfamiliar coordination to known activities).

Freehand bimanual multijoints coordination demonstrated by artists and athletes in dancing, gymnastics and Martial arts etc. reflect

${ }^{\star}$ Correspondence to: Yong $\mathrm{Li}$, Neurologische Klinik und Poliklinik, Klinikum rechts der Isar, Technische Universität München, Ismaninger Str. 22, 81675 Munich, Germany, E-mail: yong.li@lrz.tu-muenchen.de

Key words: bimanual multijoints coordination, constraint, motor control, interlimb, intralimb, inter-hemispheric, intra-hemispheric interactions

Received: June 06, 2018; Accepted: June 14, 2018; Published: June 16, 2018 
genuine principles and characteristics of human movements. This drives us to be the first to explore the principles behind the challenging but exciting bimanual multijoints coordination movements [15-17]. The scientific motivation to explore bimanual multijoints coordination in freehand came from a question: neuromuscular constraints governs bimanual single joint coordination while directional constraints brings in stable patterns within a limb without homologous muscles, then what will happen if we perform bimanual coordination with two joints within each upper arm? Would the two domains of constraints be simply summed up or a new coalition of constraints would emerge to master this more challenging task? To tackle the coalition of constraints in human bimanual multijoints coordination, my colleagues and I designed a series studies to systematically research bimanual motions including all 6 major joints of the upper-limbs, namely bilateral wrist, elbow and shoulder joints. Our objective was to discover what will happen when homologous muscles controlled by inter-hemispheric interactions encountered to the very segments directed by intrahemispheric intervention? We applied in-phase and anti-phase motions to bimanual joints while iso-directional and noniso-directional patterns to joints within an arm. To restrict task complexity in a manageable range, we employed a 4 bilateral upper limb joints $\mathrm{x} 3$ combinations design. Our findings not only confirmed that fundamental principles previously found in bimanual two joints coordination preserved in bimanual multijoints coordination but also discovered a hierarchical control mechanism of these fundamental principles. The most stable pattern in bimanual multijoints coordination, ININ-ISOISO, is a summation of both neuromuscular and directional constraints. Between bilateral limbs, neuromuscular constraints cooperating with mirror-symmetry (egocentric) powerfully master bimanual multijoints coordination system. Within each limb, directional constraints working with synergistic muscle co-activation result in another stable pattern, ANAN-ISOSIO [15-17]. This finding also fits the principle of directional compatibility in extrinsic space (underlying translational symmetry, allocentric constraint). In this hierarchical control structure of control constraints that interlimb coordination constraints played a more prominent role than that governing intralimb coordination. The relative weight of all constraints varies by the modulation of task demands, for example, cycling frequency manipulation, and other important factors, such as adjacent joint interaction within a limb, limb dominancy etc [15]. Ultimately, it is the gestalt of the whole hierarchy that constitutes such a beauty of complexity in human limb movement.

The advantage of such experimental designs is that all subtasks/ subcomponents have been validated by other researches under various conditions $[16,17]$. Non-dominant limbs can easily perform default bimanual and unimanual patterns. Such a combination in task design serves as a solid foundation of the freehand bimanual multijoints coordination studies. The three studies open a new and unique window into the nature of interlimb and intralimb coordination constraints during complex bimanual multijoints coordinative performance. Our endeavors are to push bimanual coordination research systematically towards its limits with a well-controlled balance between the capability of data mining and data analysis, and actual performance ability of young volunteers recruited in these studies [15-17].

Functional brain network in bimanual coordination: Bimanual whole-hand-fist movement results in differential facilitation and inhibition of neural activity in motor areas within both hemispheres [22]. The visually paced bimanual movements increase connectivity not only related to the supplementary motor area (SMA) but also to interhemispheric primary motor cortex (M1) to M1 and premotor cortex
(PMC) connections. SMA acts to promote or suppress M1 [22]. Many other imaging studies using bilateral two-single-joints finger [23-31] or forearm movements [31-33] show that bimanual coordination evokes similar levels activation in sensorimotor network as unilateral motion tasks. Increased recruitment of neural resources in a parieto-frontal and subcortical network have been found specifically during bimanual tasks $[30,32,34]$. These bimanual coordination specific networks show contextual oriented dynamic feature depending on individual expertise level, age, and pathology as well as environmental factors, such as task difficulty and complexity. And its evoked activations may expand into frontal, parieto-occipital, temporal areas and the insular cortex. On the one hand, increasing task difficulty by elevating cycling frequency profoundly results in enhanced harmonic activation in premotor, SMA and M1 of motor area $[35,36]$. On the other hand, activations in parieto-temporal regions are required besides sensorimotor network in order to handle increased task complexity during performing nonpreferred/non-familiar coordination patterns [35]. The execution of anti-phase as compared to in-phase coordination patterns is associated with increased activity of the cingulate motor area (CMA) and its effective connectivity with the cerebellum [33]. Aging results in higher recruitment of functional activity in action planning as well as the taskrelated brain network in bimanual coordination execution [36,37]. Experience and expertise show lower degrees of neural recruitment in bimanual coordination tasks than novices $[25,38]$. Bimanual coordinative training and experience result in complex dynamic network changes by elevating plasticity within- as well as betweenhemisphere interactions $[39,40]$, thus strengthens interaction among different brain regions [41]. Bimanual coordination tasks such as handarm bimanual intensive therapy (HABIT) $[42,43]$ has shown motor improvements for the rehabilitation of children with hemiparesis $(\mathrm{CH})$. However, measurable neuroplasticity change following HABIT in $\mathrm{CH}$ that shifts to a more unilateral brain activation pattern is consistently associated with motor improvements [44].

Structural brain network in bimanual coordination: Diffusion weighted imaging studies reveal that better performance on bimanual coordinative tasks is usually consequences of higher structural connectivity in various sections of corpus callosum (CC), where connections of bilateral sensorimotor, parietal and occipital areas intersect. The initial skill learning ability of bimanual coordinative patterns can be predicted by the white matter organization of the anterior CC, which directly connects to areas involved in motor learning in the prefrontal cortex [45]. The recruitment of anterior and posterior CC are associated with highly skillful bimanual task training, for example comparing pianists with matched controls [10, 46-52]. Interactions between $\mathrm{CC}$ and bimanual coordination have been studied by mapping bimanual functions on distinct CC subregions considering factors, such as age, pathology and training [53]. White matter integrity in the middle $\mathrm{CC}$ is associated with bimanual and unimanual skills in $\mathrm{CH}$ following HABIT [54].

Limits and future opportunities: Up to now, it has not been possible to study freehand bimanual multijoints coordination simultaneously involving more than 4 joints of the upper limbs within a kinematic study. To my knowledge so far, no imaging study has attempted to employ this exciting but challenging paradigm to bring more complexity to this already complex brain network in imaging field. Technical and practical difficulties are obviously the biggest obstacles to make further progress. Nevertheless, obstacle is the way. I would expect that a breakthrough might be made in the near future to employ the freehand bimanual multijoints coordination to directly 
investigate inter- as well as intra-hemispheric brain connectivity at different levels within brain networks.

Highly contextual task complexity of this freehand bimanual multijoints coordination paradigm provides a rich resource of mulitjoints movement patterns covering various degrees of difficulty with possibility to adjust task complexity. These patterns are wellbalanced in design concerning lateralization, limb dominance and so on factors therefore can be easily/directly used for individualized training and rehabilitation treatment in elderlies or movement disorder patients. In stroke rehabilitation literature, systematically investigating bimanual coordination post-stroke and applying appropriate bimanual coordination training have been proposed [54]. More researches to characterize and quantify bimanual coordination for laboratory-based and real-world tasks as well as knowledge to implement scientific achievement to the realistic treatments are urged to be prioritized among researchers in motor control, cognitive neuroscience and rehabilitation science. The author would like to call for introducing test batteries of bimanual function as a general practice to assess the integrity of movement control and motor learning in movement disorders as valuable measure and rehabilitation programs supported by bimanual coordination research on various diseases as promising applications in healthcare big data projects.

\section{Conclusion}

Bimanual multijoints coordination researches delineate certain similarity to functional and structural configurations of the human brain. Specifically, interlimb/bilateral/bimanual coordination reflects inter-hemispheric interactions while intralimb/within-limb/ipsilateral coordination mirrors intra-hemispheric interactions of human brain functions and structures. Well understanding the hierarchy of constraints in motor control as well as functional and structural network interactions in neuroscience specified by bimanual mulitjoints coordination will help a broad range research on daily life activities including object interactive tasks, ergonomics, training as well as rehabilitation and treatment for patients with movement disorders, etc.

\section{References}

1. Bosga J, Meulenbroek RG, Swinnen SP (2003) Stability of inter-joint coordination during circle drawing: effects of shoulder-joint articular properties. Hum Movement Sci 22: $297-320$

2. Dounskaia N, Nogueira KG, Swinnen SP, Drummond E (2010) Limitations on coupling of bimanual movements caused by arm dominance: when the muscle homology principle fails. Journal of neurophysiology 103: 2027-2038.

3. Swinnen SP, Jardin K, Meulenbroek R (1996) Between-limb asynchronies during bimanual coordination: effects of manual dominance and attentional cueing. Neuropsychologia 34: 1203-1213.

4. Verschueren SM, Swinnen SP (2001) Dynamic position sense during a cyclical drawing movement: effects of application and withdrawal of tendon vibration. Neuropsychologia 39: 510-520.

5. Verschueren SM, Swinnen SP, Cordo PJ, Dounskaia NV (1999) Proprioceptive control of multijoint movement: bimanual circle drawing. Experimental brain research 127: 182-192.

6. Verschueren SM, Swinnen SP, Cordo PJ, Dounskaia NV (1999) Proprioceptive control of multijoint movement: unimanual circle drawing. Experimental brain research 127: 171-181.

7. Tseng YW, Scholz JP, Galloway JC (2009) The organization of intralimb and interlimb synergies in response to different joint dynamics. Exp Brain Res 193: 239-254

8. Aiken CA, Pan ZJ, Gemmert AWA (2015) Limb Dominance and Its Effects on the Benefits of Intralimb Transfer of Learning: A Visuomotor Aiming Task. J Motor Behav 47: 509-521.

9. Levin O, Wenderoth N, Steyvers M, Swinnen SP (2003) Directional invariance during loading-related modulations of muscle activity: evidence for motor equivalence. Experimental brain research 148: 62-76.
10. Steele CJ, Bailey JA, Zatorre RJ, Penhune VB (2013) Early musical training and whitematter plasticity in the corpus callosum: evidence for a sensitive period. The Journal of neuroscience: the official journal of the Society for Neuroscience 33: 1282-1290.

11. Vangheluwe S, Puttemans V, Wenderoth N, Van Baelen M, Swinnen SP, et al. (2004) Inter- and intralimb transfer of a bimanual task: generalisability of limb dissociation. Behavioural brain research 154: 535-547.

12. Wenderoth N, Puttemans V, Vangheluwe S Swinnen SP (2003) Bimanual training reduces spatial interference. J Motor Behav 35: 296-308.

13. Beets IA (2012) Active versus passive training of a complex bimanual task: is prescriptive proprioceptive information sufficient for inducing motor learning? Plos One 7: e37687.

14. Beets IA (2015) Reduced Neural Differentiation Between Feedback Conditions After Bimanual Coordination Training with and without Augmented Visual Feedback. Cereb Cortex 25: 1958-1969.

15. Li Y, Levin O, Forner-Cordero A, Ronsse R, Swinnen SP, et al. (2009) Coordination of complex bimanual multijoint movements under increasing cycling frequencies: the prevalence of mirror-image and translational symmetry. Acta Psychol (Amst) 130: 183195.

16. Li Y, Levin O, Forner-Cordero A, Swinnen SP (2005) Interactions between interlimb and intralimb coordination during the performance of bimanual multijoint movements. Exp Brain Res 163: 515-526.

17. Li Y, Levin O, Forner-Cordero A, Swinnen SP (2005) Effects of interlimb and intralimb constraints on bimanual shoulder-elbow and shoulder-wrist coordination patterns. Journal of Neurophysiology 94: 2139-2149.

18. Swinnen SP, Wenderoth N (2004) Two hands, one brain: cognitive neuroscience of bimanual skill. Trends Cogn Sci 8:18-25.

19. Buchanan JJ, Kelso JAS, Fuchs A (1996) Coordination dynamics of trajectory formation. Biological Cybernetics 74: 41-54.

20. Howard IS, Ingram JN, Kording KP, Wolpert DM (2009) Statistics of natura movements are reflected in motor errors. Journal of neurophysiology 102: 1902-1910.

21. Li Y, Levin O, Carson RG, Swinnen SP (2004) Bimanual coordination: constraints imposed by the relative timing of homologous muscle activation. Experimental brain research 156: 27-38.

22. Grefkes C1, Eickhoff SB, Nowak DA, Dafotakis M, Fink GR (2008) Dynamic intraand interhemispheric interactions during unilateral and bilateral hand movements assessed with fMRI and DCM. Neuroimage 41: 1382-1394 (Crossref)

23. Goerres GW, Samuel M, Jenkins IH, Brooks DJ (1998) Cerebral control of unimanual and bimanual movements: an H2(15)O PET study. Neuroreport 9: 3631-3638.

24. Immisch I, Waldvogel D, van Gelderen P, Hallett M (2001) The role of the medial wall and its anatomical variations for bimanual antiphase and in-phase movements. Neuroimage 14: 674-684

25. Jancke L (2000) fMRI study of bimanual coordination. Neuropsychologia 38: 164-174.

26. Nair DG, Purcott KL, Fuchs A, Steinberg F, Kelso JA, et al. (2003) Cortical and cerebellar activity of the human brain during imagined and executed unimanual and bimanual action sequences: a functional MRI study. Brain Res Cogn Brain Res 15: $250-260$.

27. Sadato N, Yonekura Y, Waki A, Yamada H, Ishii Y, et al. (1997) Role of the supplementary motor area and the right premotor cortex in the coordination of bimanual finger movements. The Journal of neuroscience: the official journal of the Society for Neuroscience 17: 9667-9674.

28. Stephan KM (1999) The role of ventral medial wall motor areas in bimanual coordination. A combined lesion and activation study. Brain: a journal of neurology 122: 351-368.

29. Stephan KM, Binkofski F, Posse S, Seitz RJ, Freund HJ, et al. (1999) Cerebral midline structures in bimanual coordination. Experimental brain research 128: 243-249.

30. Toyokura M, Muro I, Komiya T, Obara M (2002) Activation of pre-supplementary motor area (SMA) and SMA proper during unimanual and bimanual complex sequences: an analysis using functional magnetic resonance imaging. $J$ Neuroimaging 12: $172-178$.

31. Ullen F, Forssberg H, Ehrsson HH (2003) Neural networks for the coordination of the hands in time. Journal of neurophysiology 89: 1126-1135.

32. Tracy JI (2001) Cerebellar mediation of the complexity of bimanual compared to unimanual movements. Neurology 57: 1862-1869. 
33. Lin Q (2017) The Difference of Neural Networks between Bimanual Antiphase and In-Phase Upper Limb Movements: A Preliminary Functional Magnetic Resonance Imaging Study. Behav Neurol 8041962.

34. Wenderoth N, Debaere F, Sunaert S, van Hecke P, Swinnen SP (2004) Parieto-premotor areas mediate directional interference during bimanual movements. Cereb Cortex 14: 1153-1163.

35. Debaere F, Wenderoth N, Sunaert S, Van Hecke P, Swinnen SP, et al. (2004) Cerebellar and premotor function in bimanual coordination: parametric neural responses to spatiotemporal complexity and cycling frequency. Neuroimage 21: 1416-1427.

36. Goble DJ (2010) The neural control of bimanual movements in the elderly: Brain regions exhibiting age-related increases in activity, frequency-induced neural modulation, and task-specific compensatory recruitment. Hum Brain Mapp 31: 1281-1295.

37. Heitger MH (2013) Bimanual motor coordination in older adults is associated with increased functional brain connectivity--a graph-theoretical analysis. Plos One 8: e62133

38. Haslinger B (2004) Reduced recruitment of motor association areas during bimanual coordination in concert pianists. Hum Brain Mapp 22: 206-215.

39. Serrien DJ, Brown P (2003) The integration of cortical and behavioural dynamics during initial learning of a motor task. Eur J Neurosci 17: 1098-1104

40. Andres FG (1999) Functional coupling of human cortical sensorimotor areas during bimanual skill acquisition. Brain: a journal of neurology 122: 855-870.

41. Heitger MH (2012) Motor learning-induced changes in functional brain connectivity as revealed by means of graph-theoretical network analysis. Neuroimage 61: 633-650.

42. Gordon AM (2011) Bimanual training and constraint-induced movement therapy in children with hemiplegic cerebral palsy: a randomized trial. Neurorehabil Neural Repair 25: 692-702.

43. Sakzewski L (2011) Randomized trial of constraint-induced movement therapy and bimanual training on activity outcomes for children with congenital hemiplegia. Dev Med Child Neurol 53: 313-320.
44. Weinstein M (2015) Brain Plasticity following Intensive Bimanual Therapy in Children with Hemiparesis: Preliminary Evidence. Neural Plast 798481.

45. Sisti HM (2012) Microstructural organization of corpus callosum projections to prefrontal cortex predicts bimanual motor learning. Learn Mem 19: 351-357.

46. Schmithorst VJ, Wilke M (2002) Differences in white matter architecture between musicians and non-musicians: a diffusion tensor imaging study. Neuroscience letters 321: $57-60$.

47. Bengtsson SL (2005) Extensive piano practicing has regionally specific effects on white matter development. Nat Neurosci 8: 1148-1150.

48. Imfeld A, Oechslin MS, Meyer M, Loenneker T, Jancke L (2009) White matter plasticity in the corticospinal tract of musicians: a diffusion tensor imaging study. Neuroimage 46: 600-607.

49. Lee DJ, Chen Y, Schlaug G (2003) Corpus callosum: musician and gender effects. Neuroreport 14: 205-209.

50. Ozturk AH, Tascioglu B, Aktekin M, Kurtoglu Z, Erden I (2002) Morphometric comparison of the human corpus callosum in professional musicians and non-musicians by using in vivo magnetic resonance imaging. J Neuroradiol 29: 29-34.

51. Schlaug G, Jancke L, Huang Y, Staiger JF, Steinmetz H (1995) Increased corpus callosum size in musicians. Neuropsychologia 33: 1047-1055.

52. Wan CY, Schlaug G (2010) Music making as a tool for promoting brain plasticity across the life span. Neuroscientist 16: 566-577.

53. Gooijers J, Swinnen SP (2014) Interactions between brain structure and behavior: the corpus callosum and bimanual coordination. Neurosci Biobehav Rev 43: 1-19.

54. Kantak S, Jax S, Wittenberg G (2017) Bimanual coordination: A missing piece of arm rehabilitation after stroke. Restor Neurol Neurosci 35: 347-364.

Copyright: $@ 2018 \mathrm{Li} \mathrm{Y}$. This is an open-access article distributed under the terms of the Creative Commons Attribution License, which permits unrestricted use, distribution, and reproduction in any medium, provided the original author and source are credited. 